\title{
La fiction anticipative en France dans la deuxième moitié du XXème siècle. L'exemple de Richard Bessière.
}

\author{
Anticipative fiction in France in the second half of the 20th Century. The \\ example of Richard Bessière.
}

\author{
Jean-Louis Ermine ${ }^{1}$, Pierre Saulais ${ }^{2}$ \\ ${ }^{1}$ LITEM, Institut Mines Telecom BusinessSchool, Université Paris-Saclay, France, jean-louis.ermine@laposte.net \\ ${ }^{2}$ LITEM, Institut Mines Telecom Business School,Université Paris-Saclay, France, pierre.saulais@imt-bs.eu
}

RÉSUMÉ. La science-fiction explore les zones qui s'étendent entre la science et le mythe, la philosophie et la foi, la connaissance et le rêve. Si elle puise dans la rationalité scientifique, elle le fait toujours sous le voile de l'imaginaire. L'objet de notre contribution est d'approfondir ce cheminement d'aller et retour entre science et fiction au travers du mouvement de fiction anticipative en France dans la seconde moitié du siècle dernier, avec l'exemple de Richard Bessière, le premier et le plus emblématique auteur de la série "Anticipation ». Sur deux thématiques (économie et changement climatique) nous analysons les liens directs ou indirects entre deux espaces de pensée, celui créé par l'auteur pour son univers science-fictionnel et celui de la communauté scientifique et technique actuelle, en les inscrivant en perspective du décalage temporel qui sépare ces deux univers.

ABSTRACT. Science fiction explores the areas that lie between science and myth, philosophy and faith, knowledge and dreams. If it draws on scientific rationality, it always does so under the veil of the imaginary. The purpose of our contribution is to deepen this journey from science to fiction through the anticipatory fiction movement in France in the second half of the last century and the example of Richard Bessière, the first and the most emblematic author of the "Anticipation" series. Focusing on two themes (economics and climate change), we analyze the direct or indirect links between two spaces of thought, one created by the author for his science fiction universe and the other that of the current scientific and technical community, by placing them in the perspective of the temporal gap that separates these two universes.

MOTS-CLÉS. Science-fiction, imaginaire, innovation, fiction, anticipation.

KEYWORDS. Science fiction, imaginary, innovation, fiction, anticipation.

\section{Introduction}

Science et fiction constituent l'association étrange de la science, terrain sûr des faits expérimentés et démontrables et de la fiction, terre inconnue que seule notre imagination limite. Comme elle prend appui sur ce qu'elle sait du réel pour se projeter dans l'avenir, la science-fiction donne à voir, comme aucune autre création, le rapport de notre civilisation au progrès technique et aux avancées scientifiques.

La science-fiction explore ces zones incertaines qui s'étendent entre la science etle mythe, la philosophie et la foi, la connaissance et le rêve. Si elle puise dans la rationalité scientifique, elle le fait toujours sous le voile de l'imaginaire. N'est-ce pas en se nourrissant de la méthode scientifique que la science-fiction réinvente la notion de frontière entre la science et la fiction ? Génération après génération, les auteurs explorent cette frontière selon des axes tels que l'espace-temps, l'homme face aux machines, l'émergence d'une posthumanité et l'accélération technologique dont elle est le fruit. Ce qu'elle donne à voir, c'est tout autant une épopée humaine aux confins de l'espace et du temps, qu'un voyage intérieur jusqu'aux racines de nos imaginaires. En effet, science et science-fiction questionnent toutes deux le réel. D'un côté, la science produit des modèles et des théories décrivant notre monde et ses phénomènes, construits grâce à des expériences, des observations et des raisonnements abstraits. 
De l'autre, la science-fiction puise son inspiration dans les sciences : elle les met en scène et s'intéresse aux conséquences de leurs progrès dans le monde réel. Par-delà le noyau dur des théories établies ou de celles en cours de vérification, les scientifiques ont besoin d'un espace pour déployer leur imaginaire afin de pousser leurs théories dans leurs retranchements et élaborer des idées nouvelles, parfois irraisonnées. C'est précisément au point de rencontre où les idées sont en ébullition, aux limites entre science « acquise» et science en construction, que naît la fiction, avec ses mondes fantastiques peuplés de créatures étranges, de civilisations disparues, de technologies futuristes, mais aussi avec ses éléments de réflexion sur le monde et les enjeux du futur [COM 10].

L'objet de notre contribution est d'approfondir ce cheminement d'aller et retour entre science et fiction au travers de ce mouvement de fiction anticipative en France dans la seconde moitié du siècle dernier. Nous souhaitons ainsi analyser en quoi ce mouvement d'une part réinvente effectivement la notion de frontière entre science et fiction, d'autre part constitue un chemin de traverse vers la connaissance. Notre questionnement est donc: en quoi les auteurs représentant ce mouvement de fiction anticipative révèlent-ils une approche pertinente dans leur démarche visant à répondre à la soif des lecteurs en découvertes et en savoirs?

Nous débuterons notre analyse en situant l'historique de la fiction anticipative par ses racines temporelles exprimant soit une vision éducative illustrée par des romans de vulgarisation strictement fondés sur des hypothèses scientifiques vérifiables décalées dans un avenir très proche, soit une vision de questionnements de la science par des inventions posées à partir de théories exactes. Cet historique nous mènera à la création en France de la collection «Anticipation» des éditions Fleuve Noir, collection exceptionnelle par sa longévité, sa production pléthorique, ses succès de tirages et sa capacité d'influence auprès de lecteurs comme d'auteurs.

Le paragraphe 3 sera consacré à l'analyse de l'œuvre du premier et du plus représentatif et connu des auteurs publiés dans cette collection «Anticipation », Richard Bessière : l'exégèse de cette œuvre nous renseignera sur les rapports de l'auteur tant à la science qu'à l'anticipation, sur le vaste spectre de concepts imaginés tant dans le domaine des sciences de la matière que des sciences humaines. De cette vaste palette anticipative, nous extrairons un exemple de chacun de ces deux domaines dont l'approfondissement fera l'objet du paragraphe suivant.

La finalité du paragraphe 4, consacré à un focus illustratif sur deux thématiques (nouveaux modes d'économie de production et changement climatique) est d'explorer les liens directs ou indirects entre deux espaces de pensée, celui créé par Richard Bessière pour son univers science-fictionnel et celui de la communauté scientifique et technique actuelle. Notre analyse sera menée en trois étapes : la première étape consiste à examiner très succinctement l'état des connaissances mises en action aujourd'hui relativement aux deux thématiques. Ensuite, nous chausserons les lunettes de l'auteur pour assimiler la vision fictionnelle anticipative qu'il nous propose des deux thématiques choisies telles qu'elles apparaissent dans quatre de ses ouvrages. La troisième étape nous amènera à nous interroger sur les ponts entre l'univers de l'auteur et le nôtre actuel, en les inscrivant en perspective du décalage temporel qui sépare ces deux univers et de la part de l'imaginaire dans la capacité inventive des ingénieurs.

La conclusion de l'article sera articulée autour d'une synthèse qui dégagera les leçons apprises par notre exploration.

\section{Historique de la fiction anticipative en France}

Le terme «science-fiction » est relativement nouveau, même si on le voit apparaître dès 1851 [WIL 51]. On admet que ce terme a été pérennisé par Hugo Gernsback dans l'éditorial du premier numéro de Science Wonder Stories. Même s'il y a eu de nombreuses appellations alternatives (fiction spéculative, 
roman scientifique, etc.), le terme a fini par s'imposer. Dans le monde francophone, il s'impose à partir des années 1950, et finit par l'emporter sur d'autres termes comme « anticipation ».

L'histoire officielle de la science-fiction [BRE 12] désigne deux pères fondateurs de la sciencefiction moderne : Jules Verne (1828-1905) et Herbert George Wells (1866-1946). Il existe cependant des précurseurs. En France, les ouvrages connus ont clairement une connotation de prospective sur l'avenir, contrairement aux précurseurs anglo-saxons (Marie Shelley ou Edgar Poe par exemple) comme L'an 2440, rêve s'il en fut jamais [MER71], Le roman de l'avenir [BOD 34], Le Monde tel qu'il sera [SOU 46].

Avec ses romans scientifiques tels que De la Terre à la Lune [VER65], Autour de la Lune [VER70] ou Vingt mille lieues sous les mers [VER71], Jules Verne va ouvrir, en France, un sillon, qui ne se refermera jamais, d'une littérature foisonnante, d'« imagination scientifique » d'" anticipation scientifique », de « merveilleux scientifique », de « roman d'hypothèse », «d'aventures extraordinaires »... Pour le XIXème siècle, noosfere, le site de référence en la matière, (http://www.noosfere.org/), recense 236 livres dans ce domaine. De 1863 (première parution de Jules Verne) à 1951 (première parution de Richard Bessière), Serge Lehman, un écrivain et essayiste français, a évalué à environ trois mille le nombre de romans de «science-fiction » (selon l'appellation désormais consacrée) publiés en France [LEH 06]. On mentionne souvent les grands auteurs, qui ont été maintes fois réédités, parmi eux il y a toute une lignée d'écrivains d'anticipation scientifique comme Albert Robida, (1848-1926) auteur d'anticipation digne de Jules Verne, Maurice Renard (1875-1939), Gustave Le Rouge (18671938), H. Rosny Aîné, pseudonyme commun des frères Joseph Henri Honoré Boex (1856-1940) et Séraphin Justin François Boex (1859- 1948), Paul d’Ivoi, (1856-1915), etc.

En 1951, apparaît la collection Anticipation des éditions Fleuve Noir, avec son premier auteur qui est Richard Bessière. C'est la collection mythique de la science-fiction populaire française [AND 75]. Elle a fortement influencé toute une génération de lecteurs et d'auteurs. Cette collection est un exemple exceptionnel pour plusieurs raisons. D'abord sa longévité : elle débute en 1951 et se termine en 1997, mais ne se termine d'ailleurs pas vraiment, car elle continue durant quelques années sous d'autres formes ; elle compte exactement 2002 volumes, ce qui est un record de productivité. Ensuite son professionnalisme, puisque les auteurs vivaient de leur plume, ce qui est exceptionnel en France. Enfin son succès, puisque les tirages moyens se situaient entre 25000 et 30000 exemplaires.

La continuité avec la tradition de la science-fiction d'expression française est assurée avec la collection Anticipation, mais «dans un isolement presque total » [AND75]. En effet, avec l'arrivée en force de la science-fiction anglo-saxonne dans les années 1950, un autre sillon va se creuser. Une nouvelle génération d'auteurs français investit avec force ce nouveau créneau. En 1975, le bilan est en demi-teinte, Jacques Sadoul, dans son Histoire de la Science-fiction Moderne [SAD 75], dit que durant la période 1962-1975, la science-fiction française s'est complètement effondrée, après le feu de paille de la décennie précédente. Il déclare que dans tout ce tourbillon, la collection Anticipation du Fleuve Noir « semble avoir enfin réussi à créer une école de science-fiction française ». D'après les données récentes sur la production dans ce domaine [ERM 16], il semble que, en France, on est loin d'une mort annoncée de la science-fiction, comme certains le présagent [PRO 14].

La collection Anticipation est, comme son nom l'indique, une collection de science-fiction tournée vers l'anticipation scientifique, héritière de la lignée de Jules Verne et des auteurs cités ci-dessus. Elle est extrêmement significative de la science technologique de son époque, de ses ambitions, de son esprit. Elle s'en est très largement inspirée, et l'a projetée, grâce à l'imagination de ses auteurs, dans des dimensions prospectives qui se sont parfois avérées justifiées, sinon exactes, comme on le verra dans l'exemple traité dans cet article.

Le premier auteur de cette collection est Richard Bessière, caractéristique à la fois de cette époque et de cet esprit. Richard Bessière publiera 98 livres dans la collection Anticipation de 1951 à 1985. 


\section{Richard Bessière et l'anticipation}

\subsection{Richard Bessière et la collection Anticipation des éditions Fleuve Noir}

On connaît Richard Bessière par quelques études sur son œuvre, et par une autobiographie qu'il a rédigée quelques années avant sa disparition [BES 05]. Richard Bessière a vécu à Béziers de 1923 à 2011.

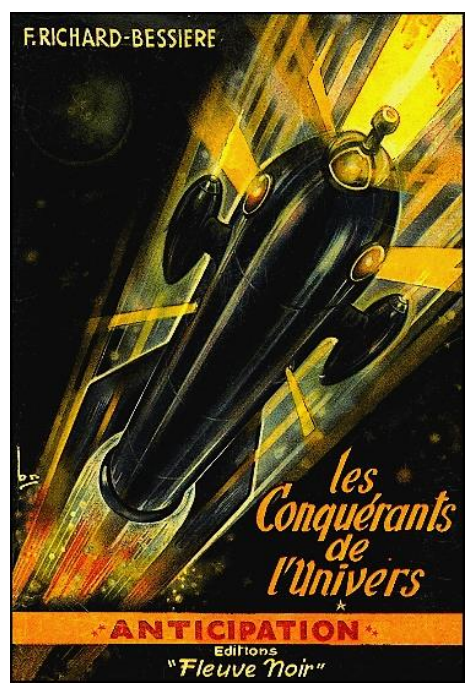

Figure 1. Exemple de couverture (Source : [ERM 16])

Écrivain populaire prolifique, il a publié plus de 220 livres dans de nombreux genres : espionnage, historique, policier, ésotérisme, la science-fiction restant son domaine préféré, pour lequel il publiera une centaine d'ouvrages, essentiellement aux éditions Fleuve Noir. C'est un écrivain phare de ce type de science-fiction, puisqu'il a inauguré la collection Anticipation des éditions Fleuve Noir avec une saga mythique d'aventures dans l'espace, naïve et iconoclaste : Les conquérants de l'Univers en 5 volumes. Cette collection a été un point marquant et pérenne dans l'émergence d'un nouveau type de science-fiction en France, qui marque un tournant général dans cette littérature et Richard Bessière est un élément caractéristique de ce tournant.

Héritier déclaré de Jules Verne, Herbert George Wells et Edgar Rice Burroughs mais aussi d'auteurs francophones comme René Barjavel, J.-H. Rosny Aîné, Jean de la Hire, etc., c'est dans la lignée de cette culture que Richard Bessière va inscrire son œuvre de science-fiction, mêlant de manière disparate et incongrue des idées naissantes sur des nouveautés techniques ou scientifiques à peine découvertes, en même temps que des stéréotypes de héros à l'ancienne ou des clichés d'aventuriers intrépides.

Curieux de tout, il s'est informé sur d'innombrables sujets touchant aux sciences de toutes sortes : sciences dures comme les mathématiques, la physique, la biologie, etc., sciences humaines comme la sociologie, la psychologie, la philosophie, la théologie, l'économie, etc., jusqu'aux sciences occultes comme la théosophie, le spiritisme, l'alchimie, l'occultisme, etc. Il en a fait grandement profiter ses lecteurs qui peuvent, à travers ses livres, trouver de multiples références à ces domaines. Richard Bessière a contribué de manière originale à tous les thèmes maintenant bien connus de la sciencefiction : Les envahisseurs extraterrestres, les guerres interplanétaires, les voyages temporels, les mondes parallèles, les espèces xénogènes, les dangers de l'espace et les planètes inhospitalières, la Terre dystopique, les mondes subatomiques ou subterrestres. Il a beaucoup innové dans le domaine en écrivant sur des thèmes liés aux sciences occultes - ce qui était nouveau à l'époque - (les civilisations disparues ou anciennes, les continents légendaires), aux spiritismes (le monde des esprits) et à la limite des neurosciences (le transfert psychique). 


\subsection{Richard Bessière et la science}

Dévorant des magazines dits de vulgarisation scientifique comme Cosmos, Sciences et Vie, Planète, etc. il avait une vue assez large, mais évidemment superficielle, sur les avancées scientifiques de son époque et un vocabulaire conséquent dans le domaine. Ce qu'il en a fait mérite d'être souligné. Ce vernis scientifique a été pour lui une source d'inspiration incroyable. Il a su jongler avec les mots, les concepts, les idées. Il a eu des intuitions surprenantes, qui ont émergé de cette accumulation un peu chaotique. Il a bâti des constructions intellectuelles étonnantes qui avaient l'air rationnelles, mais qui étaient en fait totalement irréalistes, voire surréalistes.

Presque aucune de ses constructions sur la science ne résiste à la moindre analyse. Si certaines de ses idées sont quand même visionnaires (certaines sont évoquées dans le $\S 3.3$ ), elles ne reposent sur rien de sérieux. Il semblait manquer d'esprit critique vis-à-vis de ce qu'il écrivait et surestimait sa compétence scientifique. Mais à l'époque, on dissertait sur les écrivains de science-fiction comme précurseurs de la science «tout court» et l'exemple de Jules Verne (qui a pourtant proféré beaucoup d'inepties) est quand même dans tous les esprits comme celui d'un écrivain d'anticipation de premier ordre. Il est maintenant clair qu'il y a deux professions distinctes : celle de scientifique (on parle de « chercheur scientifique $\gg)$ et celle d'écrivain de science-fiction. Il est assez courant que les deux s'apprécient, mais plus personne ne mélange les genres. D'un côté, il y a le rationalisme et la méthode (assez desséchants, il faut bien l'avouer) réservés à un petit nombre et, de l'autre, il y a l'art et le rêve (qui donnent du plaisir au plus grand nombre), même s'il y a une frontière très poreuse entre les domaines de la science et de l'imaginaire.

\subsection{Les anticipations de Richard Bessière}

L'œuvre de science-fiction de Richard Bessière est truffée jusqu'à plus soif de références scientifiques, d'explications rationnelles (qui n'en sont pas vraiment...), de dispositifs imaginaires incroyables basés sur des théories savantes, de discours parfois interminables concernant toutes les sciences : la physique, la chimie, la biologie, les techniques, mais aussi les sciences humaines comme la sociologie, la linguistique, voire même l'économie ou la philosophie. C'est un aspect incontournable de ses ouvrages et il mérite une analyse même partielle.

On a parlé de Richard Bessière comme du « Nostradamus du XXème siècle », mais c'est plutôt à Jules Verne qu'il faut se référer. Richard Bessière a parfaitement illustré ce qu'on attendait d'une collection nommée Anticipation en imaginant des pistes qui pourraient se réaliser dans le futur. De ce côté, il a fait preuve d'une intuition étonnante. De l'accumulation un peu confuse telle qu'on la voit dans ses descriptions " scientifiques », d'un pathos parfois peu cohérent, d'un discours généraliste et souvent scientiste a émergé pourtant un grand nombre de concepts « futuribles » (ce nouveau mot à la mode, formé de la fusion de futurs et de possibles). Citons par exemple, sans souci d'exhaustivité : la station orbitale [BES 55], le satellite espion [BES 58b], le cycle du Big Bang [BES 58], la création d'un humanoïde à partir d'une cellule [BES 59], la notion , chère au transhumanisme actuel, « d'amplification d'intelligence » [BES 60], etc. Mais l'innovation anticipative de Richard Bessière ne s'arrête pas à la technologie ou à la « science dure ». Il a remarquablement pointé des avancées dans des sciences sociales qu'on retrouve de nos jours. comme la sociologie, la sémiotique, la linguistique, l'économie etc.

On le voit, l'inventivité de Richard Bessière faisait feu de tout bois et sa capacité à imaginer des inventions qui se révèleront pertinentes dans le futur est tout à fait étonnante. D'autant plus étonnante que les longs discours scientifiques qui les étayaient souvent relèvent plus du sottisier que de la justification pertinente. En fait, en tant que romancier, il ne voulait pas faire œuvre de vulgarisation scientifique, mais plonger le lecteur dans un contexte scientifique dont il a eu le mérite d'utiliser le vocabulaire et de le faire connaître à des non initiés. 
Le lectorat de l'époque a adoré ces aventures pseudo-scientifiques. Sans aucun doute moins au courant que la génération actuelle, les lecteurs de Richard Bessière ont accordé peu d'attention à la crédibilité du discours, préférant sa capacité à porter des rêves. Encouragé par ce succès, Richard Bessière a persévéré longtemps dans cette voie, en se renseignant beaucoup, en absorbant un grand nombre d'idées et de concepts. Il en a fait des «porteurs de rêves », qu'il ne faut pas voir comme des prétentions scientifiques mais comme une invitation à partager ses parcours imaginatifs et ses propres délires. Il s'agit bien, en fait, de délires dans le sens propre d'un ensemble de convictions non fondées, auxquelles Richard Bessière n'adhère pas de façon inébranlable, mais qu'il veut utiliser pour faire croire ses lecteurs en une science qui peut tout créer et tout expliquer. Le succès qu'il a eu auprès de ces derniers peut laisser entendre que soit ces lecteurs se sont laissés impressionner, soit ils n'y attachaient pas beaucoup d'importance, soit encore ils s'en sont bien amusés (comme ce fut notre cas personnel), la fonction distrayante du genre étant un de ses grands atouts.

Nous allons maintenant détailler deux exemples très actuels sur le changement climatique et les nouveaux modes d'économie de production.

\section{Focus illustratif sur deux thématiques : nouveaux modes d'économie de production et changement climatique}

\subsection{Les rapports des récits fictionnels de Richard Bessière avec les connaissances actuelles}

La finalité de ce paragraphe est d'explorer les liens directs ou indirects entre deux espaces de pensée, celui créé par Richard Bessière pour son univers science-fictionnel et celui de la communauté scientifique et technique actuelle. Cette exploration sera limitée à deux thématiques très actuelles: les nouveaux modes d'économie de production et le changement climatique.

Le thème des nouveaux modes d'économie de production est peu abordé dans la science-fiction, c'est ici une innovation de Richard Bessière. En revanche, celui de la catastrophe climatique est vieux comme le genre lui-même, puisque déjà Jules Verne l'aborde dans [VER 89] avec une histoire d'inversion des pôles ou dans [VER83] où la ville affronte un refroidissement soudain. Ce thème est bien sûr très à la mode maintenant et on l'appelle fiction climatique, mais il apparait déjà chez des auteurs classiques, comme J-G Ballard [BAL 62a], [BAL 62b], [BAL 64] ou Brian Aldiss [ADL 62].

Sur ces deux thématiques, nous avons choisi de mener une analyse en trois étapes : la première étape, très factuelle, va commencer par un tour d'horizon très succinct de l'état des connaissances mises en action aujourd'hui relativement aux deux thématiques choisies pour leur actualité. La suite consistera à chausser les lunettes de l'auteur pour assimiler la vision fictionnelle anticipative qu'il nous propose de ces deux thématiques telles qu'elles apparaissent dans quatre de ses ouvrages. La troisième étape nous amènera à nous interroger sur les ponts entre l'univers de l'auteur et le nôtre actuel, en les inscrivant en perspective du décalage temporel qui sépare ces deux univers et de la part de l'imaginaire dans la capacité inventive des ingénieurs. Enfin, nous élargirons le débat à l'examen de l'influence que Richard Bessière a pu avoir sur son propre monde des auteurs de science-fiction.

\subsection{Les deux thématiques vues par la connaissance scientifique actuelle}

\subsubsection{Thème des nouveaux modes d'économie de production}

Nous nous proposons ici d'évoquer l'impression 3D, la diffusion numérique et la dématérialisation.

La production d'objets par duplication à faible coût peut actuellement se caractériser par deux axes de développement, l'un, physique, avec l'imprimante 3D et l'autre, virtuel, avec la diffusion numérique, notamment logicielle. 


\subsubsection{L'impression 3D}

L'impression 3D (ou fabrication additive) concerne des procédés volumiques de fabrication de pièces par ajout ou agglomération de matière. Un concepteur dessine l'objet 3D grâce à un outil de conception assistée par ordinateur (CAO). Le découpage en tranches des différentes couches nécessaires à la réalisation de la pièce est envoyé à l'imprimante qui dépose ou solidifie la matière couche par couche jusqu'à obtenir la pièce finale.

Son avantage est de réaliser une production à la demande du client. Le produit est stocké sous forme digitale puis envoyé à des services d'impression 3D locaux ou imprimé en 3D grâce à l'imprimante personnelle du consommateur [LAV 13].

Parmi les très nombreux domaines d'application de l'impression en 3D, on peut citer le bioprinting (impression de tissus vivants), le food printing, la mode et les accessoires, l'architecture, ...

\subsubsection{Diffusion numérique et dématérialisation}

Nous abordons maintenant le cadre des fichiers numériques représentant soit des données soit des programmes informatiques.

\section{Diffusion des données}

Concernant les données (fichiers audio ou vidéo, livres, jeux vidéo, ...), les avantages sont liés à la vente directe (moindre impact environnemental), à la disponibilité globale (en particulier via Internet), la facilité de production (un seul original, pas de dépôt, copie à coût quasi-nul).

\section{Diffusion des logiciels}

Concernant les logiciels, on va retrouver toutes les caractéristiques de la diffusion numérique, plus les spécificités apportées par les logiciels « libres » ou «Open source ». L'expression « logiciel libre » fait référence à la liberté pour tous d'exécuter, de copier, de distribuer, d'étudier, de modifier et d'améliorer le logiciel. Au même titre que le logiciel libre, l'Open Source participe à la mutualisation des connaissances, à la création de réseaux mondiaux et au déclin de l'âge de la propriété, en s'appuyant sur les principes suivants : «Accès aux produits et/ou aux outils», « Droit de modifier et de réutiliser», « Communication horizontale et coopération », « Auto-organisation en réseaux hétérogènes», « Acceptation de la diversité, la participation au profit de l'appartenance ».

\subsubsection{Bilan}

Nous avons pu observer l'actualité des deux axes de réalisation de ce thème et leur entrée dans notre vie quotidienne.

\subsubsection{Thème du changement climatique}

Nous souhaitons ici définir succinctement le contenu scientifique de quelques notions de base relevant du thème du changement climatique et apparaissant dans l'œuvre de Richard Bessière. Nous nous proposons ainsi d'aborder les notions d'atmosphère, d'ozone et d'effet de serre.

\subsubsection{L'ozone}

\section{L'atmosphère}

L'atmosphère est une enveloppe gazeuse extrêmement mince entourant notre planète, qui, sans cela, serait une boule rocheuse sans vie : c'est l'atmosphère qui fournit aux êtres vivants des éléments essentiels tels que le carbone, l'azote et l'oxygène et qui maintient la température nécessaire à la survie des espèces. À la fois écran solaire et isolant thermique, les couches d'air qui entourent notre planète protègent la vie contre les rayons du Soleil tout en retenant la chaleur qui, sinon, s'échapperait dans l'espace pendant la nuit [HAU 07]. 
Le profil vertical de l'atmosphère fait apparaitre plusieurs zones. La troposphère est la partie basse de l'atmosphère (allant du sol jusqu'à environ $10 \mathrm{~km}$. Au-dessus s'étend la stratosphère, de $10 \mathrm{~km}$ à 50 $\mathrm{km}$ au-dessus du sol. L'ozone va intervenir par ses propriétés optiques (aptitude à absorber le rayonnement solaire ultraviolet mais également le rayonnement terrestre infrarouge) et chimiques (création ou destruction).

\section{Création de l'ozone stratosphérique}

La création de l'ozone par photolyse agit comme un bouclier protecteur filtrant les ultraviolets.

\section{Destruction de l'ozone stratosphérique}

Certains gaz, actifs chimiquement, vont contribuer à la destruction des molécules d'ozone : le méthane, le protoxyde d'azote ou des halocarbonés. Au travers de nos activités industrielles et agricoles, nous émettons en quantité toujours plus grande de nombreux polluants et alimentons indirectement la stratosphère en radicaux destructeurs de l'ozone.

\section{L'ozone troposphérique}

Dans la troposphère, l'ozone provient essentiellement de réactions chimiques avec les polluants émis par les activités humaines, en particulier les oxydes d'azote, le monoxyde de carbone et les hydrocarbures. La capacité de l'ozone troposphérique à absorber le rayonnement terrestre infrarouge fait de lui un puissant gaz à effet de serre participant au réchauffement climatique.

\subsubsection{L'effet de serre}

\section{Qu'est que l'effet de serre?}

L'énergie solaire nous arrive sous forme de lumière : la plus grande partie chauffe directement la surface de la Terre. En retour, celle-ci rayonne sa chaleur vers l'espace sous forme de rayonnement infrarouge. Mais certains gaz sont capables d'absorber ce rayonnement et de le ré-émettre vers la surface, induisant un chauffage additionnel de celle-ci. Sans cet effet, sur les surfaces continentales, les soirées seraient glaciales, même l'été. Et la température moyenne à la surface de la Terre serait de -18 $\mathrm{C}^{\circ}$, au lieu de $+15 \mathrm{C}^{\circ}$ ! L'atmosphère terrestre alimente donc ce mécanisme appelé « effet de serre », qui est, à l'origine, un phénomène naturel.

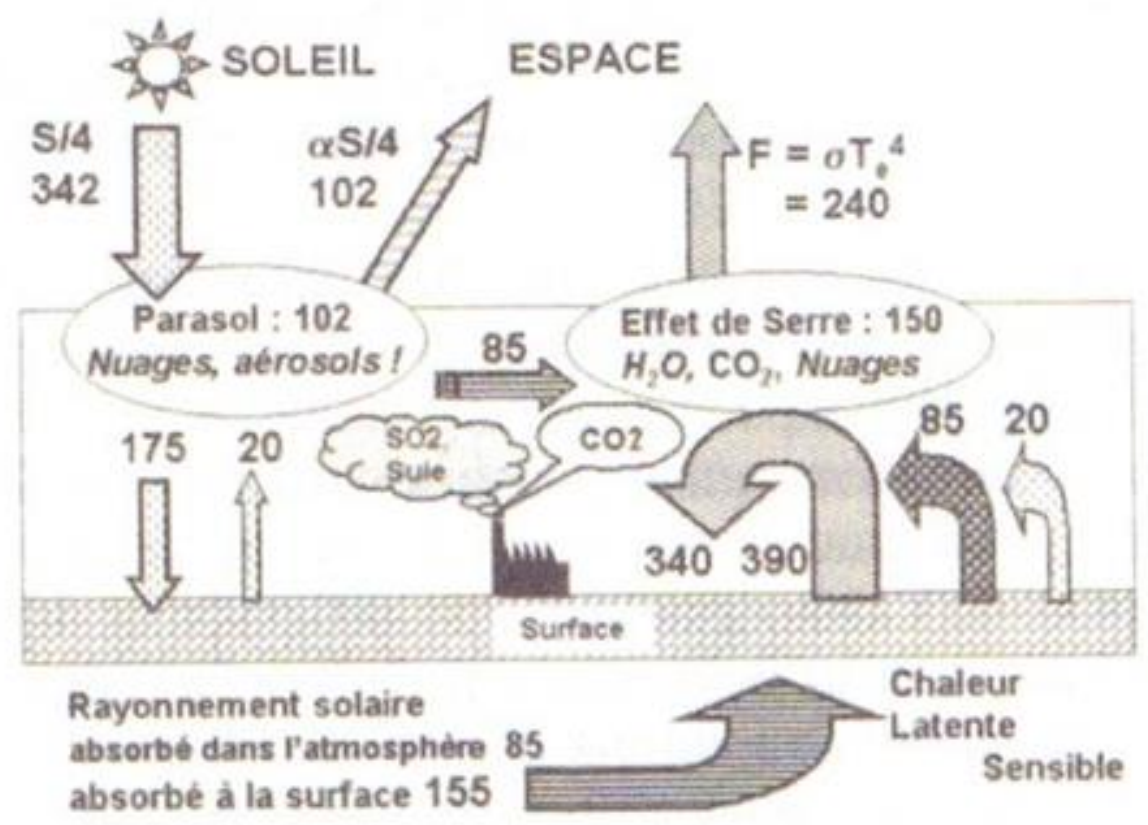

Figure 2. Transformation de l'énergie solaire (Source : [KAN 02]) 
La combustion des carburants fossiles (charbon, pétrole) relâche du gaz carbonique dans l'atmosphère. Le méthane provient de divers processus de fermentation à l'abri de l'air (rizières, ruminants, décharges). L'oxyde nitreux s'accumule également, pour cause d'usage massif des engrais.

\section{Quelles sont les conséquences de l'excès d'effet de serre pour le climat?}

L'augmentation de l'effet de serre a pour conséquence immédiate d'échauffer les basses couches de l'atmosphère, ce qui pourrait entraîner une augmentation du nombre des ouragans (se formant quand la température de l'océan dépasse $27{ }^{\circ} \mathrm{C}$ ). Ailleurs, les eaux océaniques plus chaudes pourraient s'évaporer davantage et provoquer des tempêtes plus intenses sur la terre proche. Lorsque la mer se réchauffe, l'eau se dilate et le niveau augmente.

\subsection{La fiction anticipative de Richard Bessière dans le cadre des deux thématiques choisies}

\subsubsection{Description de quatre œuvres de Richard Bessière}

Dans ce paragraphe, nous décrivons brièvement des romans de Richard Bessière qui abordent les deux thèmes de notre étude : les nouveaux modes d'économie de production et le changement climatique.

-Objectif Soleil [BES 56] Sur une trame d'espionnage entre américains et russes, c'est l'histoire d'une planète qui dépérit, car sa couche d'ozone s'épaissit et la planète refroidit irrémédiablement. Des bouleversements écologiques, notamment microbiens, se produisent. Les habitants de cette planète veulent augmenter l'activité solaire et réchauffer leur planète. Mais ceci menace bien sûr la Terre, dont il faudrait par antagonisme augmenter la couche d'ozone !

- Réaction déluge [BES 59] On découvre une race extraterrestre qui estime que la race terrienne est incapable d'atteindre un stade suffisant de connaissances. Afin de rebâtir un nouveau monde, elle veut détruire la race terrienne en déclenchant d'immenses cataclysmes sur la planète. Pour cela, elle veut injecter des milliards de tonnes d'anhydride de carbone dans l'atmosphère terrestre, afin d'augmenter considérablement le réchauffement de la planète, la montée du niveau des océans, etc.

-Terre degré 0 [BES 60] Des savants géniaux conçoivent des moteurs sans combustible, des aliments inépuisables et économiques, ils envisagent d'instaurer un nouvel ordre social pour éradiquer les mauvais penchants de l'humanité. Une bombe russe explose dans la stratosphère, créant un phénomène météorologique désastreux: la couche d'ozone de l'atmosphère se met à augmenter, empêchant le soleil de réchauffer la Terre et ainsi s'annonce une glaciation rapide et destructrice.

-Plus égale moins [BES 61] Des phénomènes étranges se répandent : les marchés sont inondés de produits à bas prix, sur lesquels tout le monde se rue. La bourse de New York commence à s'affoler, car on peut tout acheter sans payer le coût de production. On y voit un lien entre la disparition de matière dans les différents coins du monde et la production sans coût d'objets. En fait, c'est un homme d'affaires qui a ramené un extraterrestre échoué sur la Terre et possédant la faculté de dupliquer les objets à l'infini, à partir de n'importe quelle matière. L'aspect «économie fiction» du roman est très développé. Il y a de nombreuses discussions et polémiques sur cette économie d'abondance, où tout bien de consommation se produirait à coût marginal, où il n'y aurait donc plus besoin de travailler et tout serait disponible pour tous, mais ce modèle économique pourrait être plein de danger pour l'humanité.

\subsubsection{La sphère scientifique et fictionnelle de Richard Bessière illustrée par Objectif Soleil}

Dans Objectif Soleil [BES 56], Richard Bessière imagine des phénomènes liés à la couche d'ozone des planètes (la planète Gota et la planète Terre). Le rôle de la couche d'ozone est connu : intercepter les radiations néfastes en provenance du soleil (p. 83). Le dérèglement de la couche qui s'épaissit sur 
Gota met en danger son humanité (ibid.), avec les risques d'accroissement de la superficie des calottes polaires, de développement de la gent microbienne, des bactéries (p. 84).

Cet environnement scientifique sert deux fois dans le roman. La solution préconisée pour sauver la planète Gota est d'augmenter l'activité de fusion du soleil (!) pour diminuer la couche d'ozone et accroître la chaleur sur cette planète. Comme cela doit causer un réchauffement climatique intense sur la Terre, la solution va consister à augmenter la couche d'ozone sur la Terre, qui produirait les effets inverses de refroidissement du climat. On peut donc dire que la sphère scientifique de Richard Bessière est le bouleversement climatique, bien connu et très en vue de nos jours, qu'il utilise dans sa fiction de deux manières différentes : refroidissement et réchauffement.

Quelques points amusants et bien anticipés sur notre réalité actuelle apparaissent aussi dans ce roman comme les «climato-sceptiques » de la planète Gota pour qui on "exagère un peu les dangers que court la Terre. Quelques degrés de plus ne risquent pas forcément de brûler votre globe » (p. 86)!

\subsubsection{La sphère scientifique et fictionnelle de Richard Bessière illustrée par Réaction déluge}

Dans Réaction déluge [BES 59], Richard Bessière aborde les deux thématiques analysées ici.

Sur la duplication à coût minimal, il critique violemment l'économie mécanisée (p. 25), qui engendre des coûts dus à la mécanisation elle-même et aux produits obsolescents qu'elle engendre : les coûts de production seraient donc réduits par la conception de produits durables, voire éternels !

Sur le changement climatique, c'est l'intervention du professeur Vikroz qui va déclencher une catastrophe climatique, en doublant la quantité d'anhydride carbonique contenue dans l'atmosphère afin d'absorber le rayonnement infra-rouge produit par le sol. Le réchauffement serait alors rapide, les glaces fondraient, élevant le niveau des mers et noyant les nations terrestres (p. 56). Vikroz pense aussi à projeter dans les nuages des cristaux d'iodure d'argent, pour décupler le volume des eaux précipitées et faciliter la destruction de la Terre!

\subsubsection{La sphère scientifique et fictionnelle de Richard Bessière illustrée par Terre degré 0}

Dans Terre degré 0 [BES 60], Richard Bessière continue sa croisade contre la domination des machines et ce qu'on appelerait maintenant «l'Intelligence Artificielle». Un savant génial, découvre un sérum «amplificateur d'intelligence » qui lui fait faire des découvertes extraordinaires, alors que l'humanité est sur la pente d'un déclin rapide et inéluctable.

Sur le thème économique, les solutions sont toujours des inventions qui fonctionnent à coût marginal, mais qui posent de vrais problèmes sociaux et économiques : des moteurs fonctionnant sans combustible (ce qui engage une vraie polémique sur le bouleversement économique engendré, p. 68), un sérum très simple à fabriquer guérissant toutes les maladies connues et inconnues (même polémique sur les conséquences, p. 70), un aliment complet, facile à produire, permettant de nourrir toute la planète.

Mais l'autre thème du changement climatique apparaît par ailleurs, car une catastrophe déclenche l'augmentation de la couche d'ozone, avec des éléments assez proches d'Objectif Soleil. Richard Bessière réutilise ses mêmes connaissances sur le domaine. La solution viendra d'un «transmutateur » dissociant les molécules d'ozone pour recomposer l'oxygène initial.

\subsubsection{La sphère scientifique et fictionnelle de Richard Bessière illustrée par Plus égale moins}

Ce roman[BES 61], sous des dehors légers, est une véritable étude socio-économique sur une mutation technologique (ici arrivant par un artefact de science-fiction) qui pourrait bien se produire dans notre futur proche. Le monde est inondé de produits de toutes sortes à coût presque nul. Ceci déclenche une folie acheteuse des consommateurs, puis une panique des groupements professionnels, 
des syndicats, des boursiers et des économistes du pays. Les objets ne sont fabriqués par aucune usine, mais par un extraterrestre qui a la faculté de dupliquer à l'infini n'importe quoi, à partir de n'importe quel matériau brut.

A cette époque (1960), comme Richard Bessière le rappelle, les projections économiques prédisaient l'instauration d'une économie prenant en considération les richesses naturelles de notre planète au profit de tous, sans pour cela envisager surproduction et chômage, ceci basé sur une nouvelle révolution industrielle, avec de nouvelles structures sociales un travail réduit une large diffusion de la culture et de l'enseignement, de gigantesques investissements intellectuels, le tout adapté à une nouvelle structure industrielle et sociale (p. 61). C'est angélique, mais c'est bien la tendance actuelle, même si les résultats escomptés ne sont pas là !

Cependant, cette révolution pointe que la suppression de la fabrication fait disparaître presque un tiers de la production nationale (p.134). On se croirait dans la nouvelle économie actuelle : Richard Bessière imagine d'ailleurs très bien le paradoxe qui se produit actuellement avec ce qu'on appelle l'économie des plate-formes (Amazon, Booking, RB\&B, Uber....).

Les luttes économiques et politiques font rage pour s'approprier ce nouvel eldorado économique, tant du côté des entreprises (un industriel véreux pilote cette révolution), que du gouvernement américain (qui veut s'approprier l'extra-terrestre).

Richard Bessière témoigne dans ce roman d'une connaissance fine des mécanismes économiques et de leurs implications sociales et politiques. Son imagination science-fictionnelle lui permet de décrire un monde où l'économie est bouleversée par une véritable révolution touchant la production de masse. Il était sûrement loin de se douter que c'est ce que nous vivrions soixante ans plus tard !

\subsection{De la fiction anticipative à la réalité actuelle : liens entre les deux univers dans le cadre des deux thématiques choisies}

\subsubsection{Thème des nouveaux modes d'économie de production}

Dans Réaction Déluge [BES 59], le professeur Vikroz considère que la civilisation industrielle (terrienne) relève d'une ancienne économie industrielle, qualifiée de "technocratie sans avenir" et qui a amené un chômage de masse par le remplacement de l'homme par les machines. Ces machines de l'ancienne économie exigent des efforts considérables pour être conçues, fabriquées, entretenues, réparées puis remplacées quand elles sont usées, alors que la nouvelle économie industrielle prônée par le professeur serait caractérisée par la visée et l'atteinte d'une perfection technique ne nécessitant ni entretien ni remplacement. Cette visée de perfection technique sans usure est aussi celle du professeur Stewart, dans Terre degré zéro [BES 60], qui a inventé des panacées pour satisfaire tous les besoins matériels humains (un sérum unique universel pour soigner toutes les maladies, un aliment unique universel pour nourrir tous les hommes, ...) sans machines. Cette vision du héros qui veut faire le bonheur des hommes sans leur demander leur avis a nourri la littérature depuis des siècles et elle reste toujours d'actualité aujourd'hui. Finalement, toutes ces aventures héroïques, menées au nom d'une libération de l'homme (en le libérant en particulier du travail et des machines), ne font que l'asservir davantage en le privant de son autonomie et en le mettant sous la dépendance de ceux qui lui fourniraient ces panacées à ses besoins matériels : c'est une approche plus politique qu'économique, largement reprise dans [HAR 66]. Concernant la vision de libération de l'homme de la machine, la réalité aujourd'hui est toute autre, puisque, dans tous les domaines, la tendance est à la transformation de l'homme en assisté, aussi bien par les machines que par les outils numériques.

Plus égale moins [BES 61] dépeint une approche beaucoup plus économique (surtout macroéconomique), quant au contexte de l'aventure de cet épisode. La nouvelle technique de fabrication qui tendrait à éliminer toute main-d'œuvre sur quantité d'articles et de produits vendus à des prix imbattables, en réduisant la durée du travail, préfigure l'avènement à la fois de la civilisation des 
loisirs, des techniques numériques de duplication en pleine expansion, de la surproduction, de la délocalisation des industries vers des pays à très faible coût de main d'œuvre, du chômage de masse et de graves crises du système économique.

Du point de vue de la production, les liens entre l'univers anticipé et l'univers actuel sont d'abord exprimés par le bouleversement des techniques de fabrication par l'automatisme sans qu'il soit permis d'envisager la ruine des espérances humaines ([BES 61], p. 136). Ces bouleversements ne constituent pas une idée nouvelle, mais le plus intéressant n'est-il pas qu'ils ne conduisent pas à la ruine des espérances ? La diffusion numérique (fichiers de textes, de musiques, de films, de jeux) apporte plutôt le bon côté d'une démocratisation culturelle, comme le fait l'esprit lié aux logiciels ouverts. Ces liens sont ensuite exprimés par la nécessaire existence d'un modèle préalablement à toute reproduction ([BES 61], p. 136), ce dont le principe de l'impression 3D est bien sûr une réminiscence. D'une certaine manière, la production sans fabrication anticipe également sur l'économie virtuelle où la valeur en bourse n'est plus en rapport avec la valeur des actifs matériels et sur la crise des subprimes [STA 15].

Au total, nous pouvons noter, à partir de l'univers de l'auteur, un foisonnement d'idées qui résonnent énormément aujourd'hui.

\subsubsection{Thème du changement climatique}

L'épisode Réaction Déluge [BES 59]s'inscrit dans la réalité actuelle, en s'appuyant sur l'effet de serre pour créer un échauffement de température par augmentation de la concentration en gaz à effet de serre. Un risque bien actuel est que l'augmentation de température qui en résulterait ferait fondre la glace polaire, élevant le niveau des mers de quelques dizaines de centimètres à quelques mètres et noyant des pays côtiers. L'auteur s'appuie également sur l'ensemencement des nuages, technique élaborée et appliquée pour la première fois en 1946, aux États-Unis, pour lutter contre la sécheresse qui sévissait dans la région de New York. L'iodure d'argent (mentionnée dans cet épisode) est la particule artificielle la plus communément utilisée pour cela. De la fiction à la réalité, on note ainsi une continuité de techniques (ensemencement des nuages) ou d'effets (augmentation de la concentration de gaz à effet de serre).

Autre facette du thème du changement climatique, le refroidissement climatique est imaginé dans deux épisodes (Objectif Soleil [BES 56] et Terre degré zéro [BES 60]) comme résultant d'un accroissement de la concentration en ozone stratosphérique accompagné d'une modification des propriétés du rayonnement solaire : un transfert de densité spectrale de puissance dans Terre degré zéro [BES 60]ou une baisse de l'énergie rayonnée dans Objectif Soleil [BES 56]. Mais les mécanismes imaginés par l'auteur sont peu fondés scientifiquement, ce qui restreint considérablement l'éventualité d'applications potentielles. Un autre obstacle à la capacité de stimulation de l'inventivité des ingénieurs provient de ce que l'auteur papillonne d'un problème au suivant en oubliant le précédent sans en évoquer les conséquences. Pour autant, la thématique elle-même est en plein essor : l'intégration du défi climatique dans les politiques d'innovation et l'intégration des enjeux d'innovation dans les politiques climatiques vont dans le sens d'une «politique d'innovation climatique » [TOU 17]. Le Groupe d'Experts Intergouvernemental sur l'Évolution du Climat (GIEC) a confirmé l'importance des impacts observés et a réaffirmé la nécessité à la fois de réduire les émissions de gaz à effet de serre et de chercher à s'adapter, car ces impacts vont s'accentuer d'ici la fin du siècle, avec des conséquences potentiellement négatives [IPC 14]. Une inflexion majeure s'est exprimée lors de la COP22 de Marrakech, c'est la mise en avant des " solutions » et des " innovations » pour faire face au changement climatique ou en limiter les impacts, pour réduire les émissions de gaz à effet de serre, pour se saisir d'opportunités qu'il peut aussi offrir [TOU 17]. Ces innovations témoignent d'une grande diversité de projets, où tous les secteurs sont concernés (énergie, transports, construction, agriculture et élevage, gestion de l'eau, santé, ...) [DUN 15], [FAO 13], [HAL 11]. 
Ainsi apparaît le mérite principal de l'auteur : la stimulation de la prise de conscience, par le grand public et par les autorités, de la problématique mal ou peu appréhendée à son époque et des conséquences que pourrait avoir une forte intervention humaine sur l'accélération de mécanismes naturels connus dans la physique et la chimie de l'atmosphère.

\subsubsection{La fiction anticipative est-elle en capacité d'influencer en profondeur l'activité de Recherche \& Développement?}

Nous avons vu, notamment au paragraphe 4.4.2 sur le thème du changement climatique, des points de convergence et des points de divergence entre la fiction anticipative de Richard Bessière et la réalité scientifique d'aujourd'hui : la convergence ne s'opère que dans la communalité des problématiques considérées. La divergence témoigne de l'écart entre la nature des connaissances mises en jeu dans la problématique commune (peu scientifiques pour le premier côté mais très scientifiques pour le second) et aussi entre les méthodes (superficielles et papillonnantes pour le premier côté, mais approfondies et raisonnées pour le second).

Dans [SAU 16], nous avons montré que la créativité nécessaire à la capacité inventive ne repose pas seulement sur l'imagination et qu'elle peut être très efficacement stimulée par l'utilisation des connaissances inventives existantes. De ce fait, la contribution de l'imaginaire devient une composante parmi de nombreuses autres, dans lesquelles les connaissances (et surtout les connaissances inventives) sont prépondérantes. La divergence entre l'apport de la fiction anticipative et les besoins de maitrise de la réalité scientifique d'aujourd'hui illustre parfaitement la réflexion du grand inventeur Thomas Edison «Le génie est fait de $1 \%$ d'inspiration et de $99 \%$ de transpiration ». Le travail sur l'imaginaire ne saurait contribuer que comme une fraction de ce $1 \%$ d'intuition inspirée, alors que les $99 \%$ ont pour prérequis des socles méthodologiques robustes adossés à des formalisations puissantes de raisonnement scientifique et nourris par le résultat de structuration, de codage et de synthèse de nombreuses et profondes connaissances inventives. Un exemple de structuration de connaissances inventives en patrimoine intellectuel inventif est fourni dans [SAU 16]: la représentation des connaissances comme constituant un système complexe permet de voir la création de connaissances inventives comme une évolution de ce système complexe à quoi il est possible d'adapter la théorie d'inspiration Chaotique de l'évolution par émergence, selon le modèle de la figure 3.

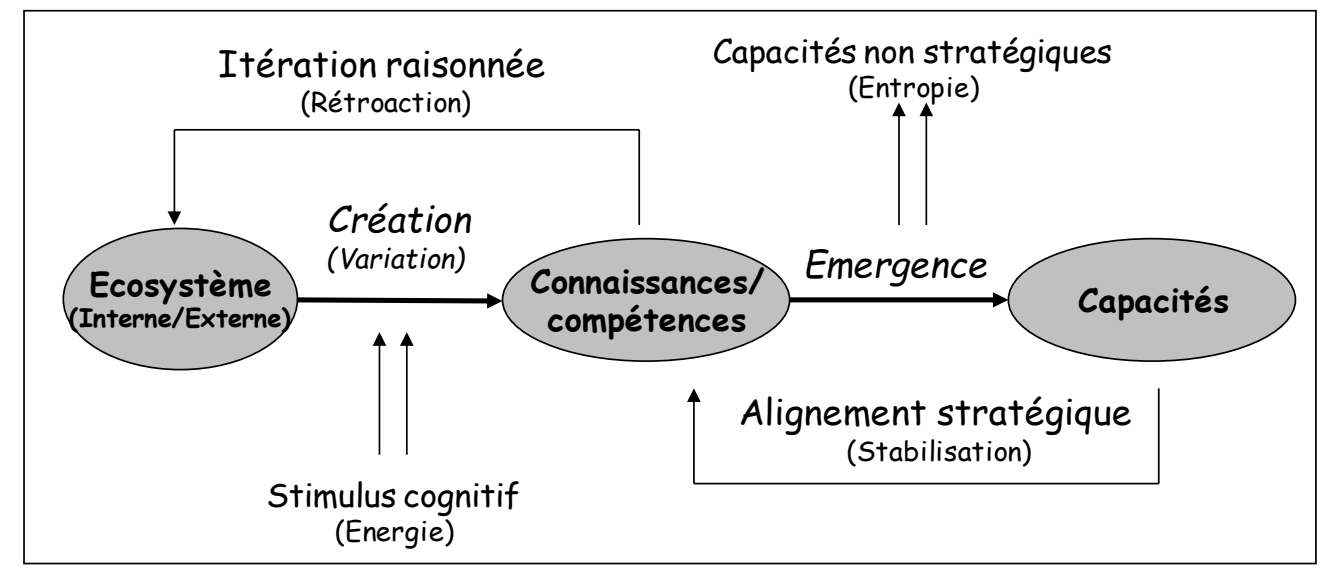

Figure 3. Le modèle «chaotique » d'évolution des connaissances par émergence (Source : [Saul 16])

Les connaissances organisées vont être enrichies sous l'effet d'un stimulus cognitif, qui résulte de l'analyse de l'existant. Ce stimulus consiste à confronter l'analyse de l'existant avec le patrimoine cognitif des acteurs de la connaissance (experts métiers), qui sont porteurs de savoirs de référence à la fois dans l'écosystème externe et interne de l'organisation. Le résultat de la confrontation va créer des variations dans les structures de connaissance, sous forme de nouvelles connaissances, soumises à une boucle de rétroaction qui va éliminer les variations qui sont trop éloignées ou trop proches des savoirs 
de référence. Il va en émerger des connaissances nouvelles qui vont être filtrées dans l'organisation par la possibilité de les mettre en action dans le cadre de sa stratégie [SAU 16].

Ainsi, le processus de création de connaissances inventives peut être représenté sous la forme d'un système dynamique non linéaire instable, non prédictible en pratique à long terme et s'appuyant sur le Deuxième principe de la Thermodynamique. Le raisonnement Chaotique voit une forte insatisfaction comme point de départ de la créativité et comme stimulus, une tension entre le désir d'évolution vers une forme supérieure d'organisation (néguentropie) et un constat d'évolution vers une forme inférieure d'organisation (entropie). Cette tension ne peut se libérer que par l'identification d'une idée source (créativité), la structuration par le raisonnement en idée d'invention, plus généralementd'œuvre (inventivité), devant se concrétiser en produit innovant [SAU 13].Ensuite, le fait de l'émergence (dont le modèle de la figure 3 utilise le principe) se manifeste en tant qu'apparition de qualités nouvelles irréductibles aux et indéductibles des propriétés des composants initiaux. Cette émergence s'applique à des connaissances inventives après un long travail de structuration qui va faire émerger des connaissances inventives nouvelles irréductibles aux connaissances inventives initiales et indéductibles de ces connaissances inventives initiales.

Dans le cadre du modèle de la figure 3, «l'imaginaire » issu de la science-fiction en tant qu'œuvre d'imagination peut venir contribuer à compléter le stimulus cognitif issu de l'analyse de la connaissance contenue dans le patrimoine intellectuel inventif. Ce stimulus enrichi va agir positivement sur l'état créatif, qui, sous certaines conditions détaillées dans [SAU 13], va engendrer une activité inventive. A notre sens, pour avoir vécu l'activité de Recherche \& Développement dans une société à très haute technologie pendant quatre décennies, la notion «d'imaginaire » chez les ingénieurs de R \& D est assez impropre et nous préférons aborder l'activité de Recherche \& Développement comme une activité inventive résultant de la combinaison d'état créatif et d'action inventive. Alors, si la fiction anticipative ne nous semble pas en capacité d'influencer en profondeur l'activité de Recherche \& Développement, nous lui reconnaissons la capacité de contribuer,parmi d'autres facteurs, à agir positivement sur l'état créatif.

Plutôt que de rechercher une influence forte de la fiction anticipative sur les ingénieurs de Recherche \& Développement, il nous semble plus approprié de rechercher si et comment la fiction anticipative de Richard Bessière a pu influencer les acteurs de son propre monde, c'est-à-dire les auteurs de science-fiction.

\subsection{Influence de Richard Bessière sur le monde de la science-fiction}

Malgré la décision de Richard Bessière d'arrêter la science-fiction en 1985, pour des raisons extralittéraires, son œuvre a perduré. Plusieurs de ses livres ont été réédités : au Fleuve Noir à partir de 1988, aux éditions J'ai Lu (1990), aux éditions Astratom (2002), aux éditions Eons (à partir de 2005), sans compter les éditions étrangères : tchèques avec United Fans, (1992), les magnifiques éditions russes que sont Canon (1995), Phlox (1994) ou le magazine Neva (1992) et les éditions américaines avec Black Coat Press, (en 2010 et 2014). Les éditions Rivière Blanche ont publié deux inédits de Richard Bessière en 2005 et 2007. Les éditions Ananké ont réédité en 2014 une magnifique version de la bande dessinée de Raoul Giordan [GIO14]. Il semble donc que le mouvement n'est pas près de s'arrêter. Plus incroyable encore, la «tradition Richard Bessière » a commencé à se perpétuer, d'abord par l'expérience originale d'écriture « à la manière de... » de J.-M. Archaimbault qui a rédigé une suite de [BES 68] intitulée Pas de pitié pour les borloks [ARC12]. Peu après, J.-M. Archaimbault a écrit une nouvelle version de N'accusez pas le ciel [BES 64] publiée sous le titre de Katorga [ARC14].

Dans la préface de l'édition américaine [BIS14] du livre Les maîtres du silence, un vibrant hommage est donné à Richard Bessière par Stephen R. Bissette : «Il y a [dans ce livre] d'autres échos et des souvenirs de Jack London, de P. Lovecraft, de Robert E. Howard, de Carl Jung et de M.C. Escher, ainsi que des éclairs d'inspiration géniale qui préfigurent Carlos Castaneda, Stephen King, et des films 
comme Project X de William Castle \& Edmund Morris (1968), Altered States (1980), déjà cité, Dreamscape de Joseph Ruben, Chuck Russell \& David Loughery (1984), Nightmare on Elm Street de Wes Craven (1984) et ses suites, The Cell de Tarsem Singh \& Mark Protosevich (2000), de séries TV telles The Outer Limits (1963-65) et Fringe (2008-2013), entre autres, ainsi que Inception de Christopher Nolan (2010), qui a véritablement intégré la thématique des «niveaux » ou « degrés » des jeux vidéo en tant qu'aspect de la logique du rêve. Nous sommes là en présence de nouveaux paradigmes du rêve et de plates-formes pour la première génération d'un public qui est composé de joueurs de jeux. Toutes ces œuvres ont contracté une dette réelle envers leurs précurseurs de la SF littéraire dont Les Maîtres du Silence ([BES 65]) de Richard Bessière. »

Quant aux lecteurs, il est clair que Richard Bessière a popularisé (plutôt que vulgarisé) auprès d'eux une certaine idée de la science, de la technologie, avec des réflexions parfois profondes sur ses implications (les lecteurs ont souvent noté son combat contre le machinisme et la dictature de la « machine intelligente »).

On peut dire, qu'à son échelle, Richard Bessière a contribué à créer un univers imaginaire, scientifique au sens large, qui a été partagé par de nombreuses personnes, scientifiques ou profanes, dans un réseau qui s'est étendu au-delà des frontières, et au-delà de son espace de temps propre.

\section{Conclusion}

Notre contribution a approfondi le cheminement d'aller et retour entre science et fiction au travers du mouvement de fiction anticipative en France dans la seconde moitié du siècle dernier, illustrée par l'exemple de l'œuvre de Richard Bessière. En observant que celle-ci prenait bien appui sur ce qu'elle savait du réel pour se projeter dans l'avenir, nous avons bien noté qu'elle donnait à voir le rapport de notre civilisation au progrès technique et aux avancées scientifiques, au travers de son exploration des zones incertaines qui s'étendent entre la science et le mythe, la philosophie et la foi, la connaissance et le rêve.

L'exploration illustrative des deux thématiques extraites de l'œuvre de Richard Bessière nous a confirmé l'inventivité de l'auteur et son étonnante capacité à imaginer des situations anticipatives qui se révèlent étonnamment modernes en 2019 , selon un foisonnement d'idées qui résonnent énormément aujourd'hui, mais sans avoir directement inspiré d'innovations dans la communauté scientifique et technique actuelle.

Pour autant, cette analyse nous conforte dans la pertinence de l'approche du mouvement de fiction anticipative en France des années 50 (et notamment de son représentant emblématique Richard Bessière) dans sa démarche qui utilise des éléments scientifiques pertinents de l'époque dans une vision futuriste qui, soixante ans après, se révèle d'une actualité brûlante.

Sans doute le lien entre l'univers science-fictionnel de l'auteur (essentiellement extrapolé à partir de nombre de connaissances vulgarisées de l'époque nourrissant son imagination débordante) et notre univers actuel est-il de nature plus subtile : ne faudrait-il pas le chercher dans la dissémination d'une vision assez prophétique d'une capacité des hommes à introduire toutes sortes de dérèglements possibles dans des mécanismes géophysiques d'origine naturelle emmenant ces derniers dans un emballement incontrôlable? Une métaphore de ce lien pourrait être la résonance du classique «billard à trois bandes ", l'auteur touchant le grand public, le grand public faisant réagir les autorités et les autorités activant les scientifiques, le tout s'étalant sur plusieurs dizaines d'années.

\section{Bibliographie}

[AIM 98] AIMEDIEU P., L'ozone stratosphérique, Paris : PUF (Que sais-je ?), 1998. 
[ALD62] ALDIS B., Le monde vert (titre original: Hot House, Édition Folio SF pour la traduction française (2009), 1962.

[AND 75] ANDREVON J-P., «En parcourant le fleuve », sous le pseudonyme d'Italo et Tomaso TOMASINI, Univers 02, Editions J'ai Lu, collection Science-fiction, $\mathrm{n}^{\circ}$ 614, 1975.

[ARC12] ARCHAIMBAULT J-M., Pas de pitié pour les borloks, Édition Rivière blanche, 2012.

[ARC14] ARCHAIMBAULT J-M., Katorga, Édition Rivière blanche, 2014.

[BAL62a] BALLARD J-G., Le Vent de nulle part,Tristram, 1962.

[BAL62b] BALLARD J-G., Le monde englouti,Tristram, 1962.

[BAL64] BALLARD J-G., Sécheresse,Tristram, 1964.

[BES 51] BESSIERE R., Les conquérants de l'Univers, Éditions Fleuve Noir, 1951.

[BES 55] BESSIERE R., SOS Terre, Éditions Fleuve Noir, 1955.

[BES 56] BESSIERE R., Objectif Soleil, Éditions Fleuve Noir, 1956.

[BES 58a] BESSIERE R., Bang !, Éditions Fleuve Noir, 1958.

[BES 58b] BESSIERE R., Fléau de l'univers, Éditions Fleuve Noir, 1958.

[BES 59a] BESSIERE R., Réaction déluge, Éditions Fleuve Noir, 1959.

[BES 59b] BESSIERE R., Panique dans le vide, Éditions Fleuve Noir, 1959.

[BES 60] BESSIERE R., Terre degré 0, Éditions Fleuve Noir, 1960.

[BES 61] BESSIERE R., Plus égale moins, Éditions Fleuve Noir, 1961.

[BES 64] BESSIERE R., N'accusez pas le ciel, Éditions Fleuve Noir, 1964.

[BES 65] BESSIERE R., Les maîtres du silence, Éditions Fleuve Noir, 1965.

[BES 68] BESSIERE R., Ne touchez pas aux borloks, Éditions Fleuve Noir, 1968.

[BES 05] BESSIERE, R. et al., Ma route semée d'étoiles, L'EEil du Sphinx, 2005.

[BIS 14] BISSETTE S. R., Richard Bessière: Maestro of Delirium, in The Masters of Silence, Hollywood Comics, 2014.

[BOD 34] BODIN F., Le roman de l'avenir, 1834.

[BRE 12] BREAN, S., La science-fiction en France, théorie et histoire d'une littérature, Presses de l'Université ParisSorbonne, 2012.

[COM 10] COMMISSAIRES D'EXPOSITION, «Science et fiction, aventures croisées », Cité des Sciences et de l'Industrie, Paris, 2010.

[DUF 00] DUFRESNE, J-J., La physique du climat, Paris, Le Pommier, 2000.

[DUN 15] DUNLAP, R., BRULLE, R., Climate Change and Society, Oxford University Press, 2015.

[ERM 16] ERMINE, J-L., Météore !, l'univers fascinant de Richard Bessière, Éditions Rivière Blanche, 2016.

[FAO 13] FAO, Climate Smart Agriculture Source Book, Rome: FAO, 2013.

[GIO14] GIORDAN R., Météore L'Intégrale, Édition Ananké, 2014.

[HAL 11] HALleGATE, S., LECOCQ, F., de PERTHUISs, C., « Designing Climate Change Adaptation Policies. An Economic Framework », Policy Research Working Paper 5568, Washington DC, World Bank.

[HAR 66] HARRISON, H., Make Room !Make Room !, Orb Books, 1966, traduit en français sous le titre Soleil vert, Paris : Presses de la Cité, 1974.

[IPC 14] IPCC, Climate Change 2014: Impacts, Adaptation and Vulnerability, Cambridge University Press, 2014.

[KAN 02] KANDEL, R., Le Réchauffement climatique, Paris : PUF (Que sais-je ?), 2002.

[LAV 13] LAVERGNE, B. \& M., L'imprimante 3D, une révolution en marche, Edition Favre, 2013.

[LEH 06] LEHMAN, S., Hypermondes perdus dans Chasseurs de chimères. L'âge d'or de la science-fiction française, Omnibus, Paris, 2006.

[MER 71] MERCIER L-S., L'an 2440, rêve s'il en fut jamais, 1771. 
[PRO 14] PROLONGEAU, H., «La SF n'a plus d'avenir ? », Télérama 3367, 23/07/2014.

[SAD 75] SADOUL, J., Histoire de la science-fiction moderne, J'ai lu, coll. Science-fiction, ${ }^{\circ}$ D67, 1975.

[SAU 13] SAULAIS, P., Application de la gestion des connaissances à la créativité des expertset à la planification de la $R \& T$ en milieu industriel de haute technologie, Thèse de doctorat, Télécom Ecole de Management, 2013.

[SAU16] SAULAIS, P., ERMINE, J-L, «Emergence d'innovation technologique incrémentale par application, à des experts, de l'analyse du patrimoine intellectuel inventif », Innovations, n 49, vol 2016/1, pp1 103-140.

[SOU 46] SOUVESTRE E., Le Monde tel qu'il sera, 1846.

[STA 15] STAUNE, J., Les clés du Futur, Paris : Plon, 2015.

[TOU 17] TOUZARD, J-M, « Innover face au changement climatique », Innovations, n 54, vol 2017/3, pp 5-13.

[VER 65] VERNE, J., De la Terre à la lune, Edition Hetzel, 1865.

[VER 70] VERNE, J., Autour de la lune, Edition Hetzel, 1870.

[VER 71] VERNE, J., Vingt mille lieues sous les mers, Edition Hetzel, 1871.

[VER 83] VERNE, J., Paris au vingtième siècle, Edition Hetzel, 1883.

[VER 89] VERNE, J., Sens dessus-dessous, Edition Hetzel, 1889.

[WIL 1851] WILSON, W., A Little Earnest Book Upon A Great Old Subject, p. 137, Darton and co, Holborn Hill, London, 1851. 